\title{
Linx
}

Revue des linguistes de l'université Paris X Nanterre

5 | 1994

La négation

\section{Sémantique des négatifs multiples}

\section{Francis Corblin}

\section{(2) OpenEdition}

\section{Journals}

Édition électronique

URL : http://journals.openedition.org/linx/1222

DOI : 10.4000/linx.1222

ISSN : 2118-9692

\section{Éditeur}

Presses universitaires de Paris Nanterre

\section{Édition imprimée}

Date de publication : 1 juin 1994

Pagination : 279-298

ISSN : 0246-8743

\section{Référence électronique}

Francis Corblin, «Sémantique des négatifs multiples », Linx [En ligne], 5 | 1994, mis en ligne le 18 juillet 2012, consulté le 03 mai 2019. URL : http://journals.openedition.org/linx/1222 ; DOI : 10.4000/ linx. 1222

Ce document a été généré automatiquement le 3 mai 2019.

Département de Sciences du langage, Université Paris Ouest 


\title{
Sémantique des négatifs multiples
}

\author{
Francis Corblin
}

1 Le problème central examiné dans cette étude est illustré par l'ambiguïté de phrases telles que (1):

(1) Personne n'aime personne

2 Dans une des ses interprétations (1) est vrai dans un monde sans amour, ce qui semble constituer, pour reprendre la formulation de May (1989), une « abrogation du principe de compositionnalité »; en substance, l'interprétation comporte moins de négations qu'il n'y a de marqueurs négatifs dans la phrase. Ainsi caractérisé, le phénomène semble renvoyer à une propriété très répandue de la négation dans les langues naturelles: il n'y a en général pas de correspondance bi-univoque entre le nombre des marqueurs négatifs observables et les opérateurs de négation associés à l'interprétation.

May suggère l'analyse suivante pour le correspondant anglais Nobody loves nobody: lorsque deux occurrences du même quantifieur monadique no (i. e. quantifiant sur des individus) sont dans le même domaine syntaxique, ils peuvent être traités de manière classique : la représentation compte alors deux négations. Mais ils peuvent également être interprétés comme un quantifieur d'un type supérieur (polyadique). Ce quantifieur polyadique NO quantifie sur des paires d'individus, et asserte que de telles paires n'existent pas, d'où l'interprétation de (1) comme monde sans amour.

Il s'agit d'une manière très ingénieuse de résoudre le problème de compositionnalité : au lieu de concéder qu'une négation est perdue, on pose qu'avec deux négations classiques on peut en construire une autre plus puissante, qui quantifie sur des paires. Le problème principal pour cette théorie, à mon avis, est qu'elle procure surtout un moyen technique de rencontrer les données, mais à un coût élevé, et sans réel pouvoir explicatif : si NO a des propriétés qui sont très différentes de la négation ordinaire, il sera difficile d'expliquer comment il «émerge » de la co-occurrence de deux négations classiques; d'autre part, cette solution est difficilement généralisable aux cas où il $\mathrm{y}$ a plus de deux occurrences d'un marqueur négatif. Si les propriétés de NO sont réductibles à celles d'un no ordinaire avec des propriétés de liage non sélectif, alors le problème de compositionnalité subsiste ${ }^{1}$. 
5 Je développerai ici une approche sémantique du problème formulée en termes de seuil de complexité sur les auto-enchâssements négatifs interprétables.

6 L'idée générale est qu'il existe une limitation dynamique qui maintient le degré de complexité des auto-enchâssements négatifs à un niveau peu élevé.

7 La conception de cette limitation emprunte à deux approches dynamiques de l'interprétation: la théorie des représentations du discours (Kamp et Reyle 1990) qui fournit le langage de représentation; la stratégie de construction des représentations utilisée dans Gabbay et Kempson (1992), qui envisage l'interprétation d'une phrase comme un processus de raisonnement en ligne traitant successivement les informations encodées dans les marqueurs lexicaux.

8 Je me limiterai ici, pour des raisons de place, à une présentation générale de l'hypothèse.

\section{Marqueurs négatifs et négation : données et problèmes}

\subsection{Représentation des phrases négatives en $\mathrm{DRT}^{2}$}

La version de la DRT introduite dans Kamp et Reyle (1990) sera utilisée comme langage formel pour la représentation des phrases négatives. La représentation de (2) est la DRS (3) :

(2) Jean n'a pas lu un article

(3)

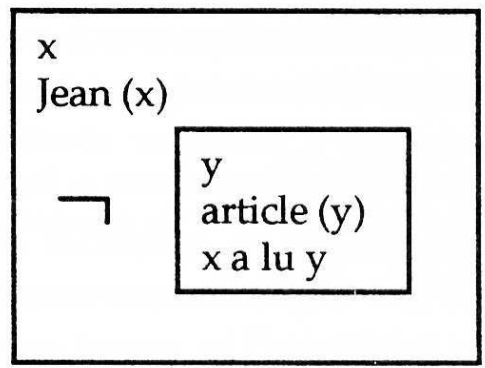

10 La DRS (3) contient une DRS principale, dont l'Univers contient le Marqueur ${ }^{3}$ de référence $x$, et deux Conditions ${ }^{4}:$ Jean $(x)$, et une Condition négative, soit une DRS précédée du symbole $\varnothing$. Cette DRS négative à son tour possède un Univers propre, contenant le Marqueur $y$, et deux Conditions. L'inscription du Marqueur de l'indéfini dans l'Univers de la DRS négative correspond à ce qu'on appelle en général interprétation d'indéfini dans la portée d'une négation. L'interprétation est: Jean n'a lu aucun article.

11 Un indéfini dans la même position syntaxique peut aussi échapper à la portée de la négation (wide-scope reading), et inscrire son Marqueur de référence dans l'Univers de la DRS principale comme en (4); l'interprétation sera alors : il y a un article que Jean n'a pas lu

(4) Jean n'a pas lu un article 


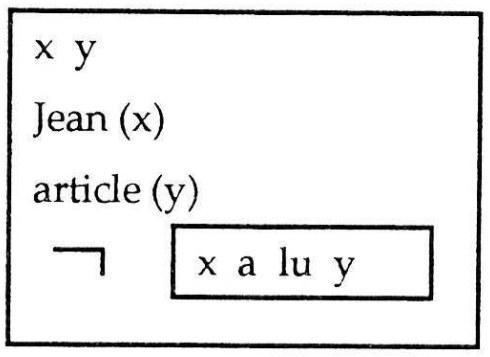

Une DRS négative étant Condition d'une DRS, l'auto-enchâssement de DRS négatives est en principe une option ouverte.

La sémantique des DRS contenant des Conditions négatives est donnée par Kamp et Reyle (1990) sous la forme d'un algorithme de vérification récursif dans un Modèle. Cet algorithme est en mesure de calculer l'interprétation de toute DRS de ce format, quelle qu'en soit la complexité. Il sera utilisé constamment dans cette étude pour déterminer l'interprétation des représentations associées aux exemples testés. Une présentation informelle de cet algorithme est donnée en (5) :

(5) Algorithme de vérification des DRS (D'après Kamp et Reyle 1990)

Soit $\mathrm{K}$ une DRS limitée au vocabulaire $\mathrm{V}$, et un ensemble $\mathrm{R}$ de Marqueurs de référence.

$\mathrm{K}$ est une paire $\left(\mathrm{U}_{\mathrm{k}}, \mathrm{Con}_{\mathrm{k}}\right)$, $\mathrm{U}$ est un sous-ensemble de $\mathrm{R}$, et $\mathrm{Con}_{\mathrm{k}}$ un ensemble de Conditions.

f est une fonction d'enchâssement de $U_{k}$ dans l'Univers $U_{M}$ d'un Modèle $M$.

1) $f$ vérifie la DRS $K$ dans $M$ ssi f vérifie chacune des Conditions de $\operatorname{Con}_{k}$.

2) f vérifie la Condition $C$ de $\operatorname{Con}_{k}$ ssi

a) $C$ est de forme $P\left(x_{1}, \ldots, x_{n}\right), P$ étant un prédicat à $n$ arguments, et $f$ projette $x_{1}, \ldots, x_{n}$ sur un n-uplet d'éléments de UM appartenant à l'extension de $\mathrm{P}$ en $\mathrm{M}$

b) C est de forme $\neg K^{\prime}$, et il n'y a pas d'enchâssement $g$ de $R$ dans $M$ qui étende $f$, et tel que $\operatorname{Dom}(\mathrm{g})=\operatorname{Dom}(\mathrm{f}) \mathrm{U}_{\mathrm{k}}{ }^{\prime}$ et $\mathrm{g}$ vérifie $\mathrm{K}^{\prime}$ en $\mathrm{M}$.

Considérons par exemple (3) : pour que $f$ vérifie cette DRS, il est nécessaire que $f$ associe à $x$ un individu $a$ de UM tel que $a$ appartienne à l'interprétation de Jean, et il faut en outre qu'aucune extension $g$ de cette fonction $f$ (e.g. aucune fonction qui associe également $x$ à $a$ ) ne projette $x$ et $y$ sur un couple d'éléments de UM qui vérifie les Conditions contenues dans la DRS négative.

Deux propriétés du langage de représentation fourni par la DRT seront d'un intérêt tout particulier :

1) sa capacité à identifier explicitement l'interprétation des phrases; de ce point de vue, un langage standard comme la logique des prédicats aurait pu être utilisé, et le sera occasionnellement ;

2) sa vocation pour les formulations dynamiques, et plus précisément la différence entre constructeurs de DRS (comme les conditionnelles, le quantificateur Universel et les négatifs) et les occupants de DRS (comme l'indéfini). A cet égard, l'usage de la logique des prédicats n'offre pas de ressources comparables, et aurait probablement un pouvoir explicatif plus faible. 


\subsection{Les marqueurs négatifs}

16 Nous appelons négation le symbole du langage formel utilisé pour la représentation sémantique des phrases négatives. En logique des prédicats, ce symbole formel est un opérateur unaire dont l'argument est une formule. En DRT, la négation est représentée par une Condition contenant une DRS, c'est-à-dire un Univers (un ensemble de Marqueurs, éventuellement vide) et un ensemble de Conditions (contenant éventuellement des DRS négatives).

17 Nous appelons négatif un item lexical de la langue naturelle déclenchant la présence d'une négation dans la représentation sémantique de la phrase. Not est un négatif dans la phrase anglaise (6) :

(6) John is not my brother

18 Une procédure effective vouée à la représentation des phrases de la langue naturelle doit minimalement disposer d'une liste des négatifs. Elle doit aussi spécifier la portée de la négation; on dira ici de choses sur cette question : l'étude se limite à des phrases simples comportant un seul verbe fini.

En français moderne, le négatif de base est (ne) $V$ pas : ne est facultatif dans beaucoup d'usages du français, notamment dans le français parlé. Nous ferons ici l'hypothèse que pas est un négatif, ne étant explétif, c'est-à-dire dépourvu de contribution propre à la construction des représentations sémantiques ${ }^{5}$. En gros, nous traitons ne comme un signal facultatif, licite si le verbe d'appui contient un négatif dans son domaine immédiat, c'est-à-dire comme une sorte d'accompagnement préverbal (facultatif) des négatifs dans le domaine du verbe ${ }^{6}$. Ce choix n'est naturellement pas indiscutable, mais le statut optionnel de ne en fait au moins une hypothèse de travail plausible.

Si une phrase détermine une représentation qui contient une négation, elle sera appelée une phrase négative. Il faut bien distinguer phrase négative et phrase positive acceptant aisément une paraphrase négative.

(7) Jean n'a pas accepté phrase négative

(8) Jean a refusé phrase positive

21 Bien que (7) et (8) soient vraies dans les mêmes mondes possibles, il y au moins une différence importante entre elles: dans la phrase négative (7) un indéfini argument du verbe peut être interprété dans la portée de la négation, alors qu'en (8), cela n'est pas possible.

(9) Jean n'a pas accepté une étudiante cette année

(10) Jean a refusé une étudiante cette année

22 (9) et (10) peuvent être synonymes, impliquant une étudiante particulière, susceptible d'être anaphorisée par un pronom singulier dans une phrase simple ultérieure du même discours. Mais seule (9) peut aussi signifier qu'aucune étudiante n'a été acceptée par Jean durant l'année. En gros, seule (9) construit une DRS négative et un Univers tel que l'indéfini puisse être un occupant de cet Univers; en (10) il n'existe pas d'Univers de ce type. La dynamique de ne pas accepter et refuser est par conséquent différente. La différence est recoupée par la distribution de aussi et non plus :

(11) Jean n'a pas accepté. Marie non plus

(12) Jean n'a pas accepté. *Marie aussi

(13) Jean a refusé *Marie non plus

(14) Jean a refusé Marie aussi 


\begin{tabular}{|l|l|l|}
\hline$(23)$ & Négatifs : & $\begin{array}{l}\text { pas } \\
\text { not }\end{array}$ \\
\hline$(24)$ & Négatifs d'Univers & $\begin{array}{l}\text { rien, personne, aucun N } \\
\text { nobody, nothing... }\end{array}$ \\
\hline
\end{tabular}

31 La particularité des items appelés négatifs d'Univers est essentiellement qu'ils déclenchent deux choses à la fois : la représentation du verbe principal comme noyau d'une Condition négative, et l'introduction d'un Marqueur de référence dans l'Univers de cette DRS négative. Ce sont en somme des constructeurs-occupants de DRS, alors que les négatifs sont uniquement des constructeurs et les indéfinis seulement des occupants de DRS.

32 Il me semble que l'analyse de ces items est plus directe en DRT qu'en logique des prédicats. D'abord, la conjonction sur un seul et même item de ces deux rôles est plus 
naturelle si on conçoit ces rôles comme distincts et complémentaires, que si on les identifie l'un et l'autre à des quantificateurs: en logique en prédicats, nous serions amenés à dire que le même item s'interprète comme une négation et un quantificateur existentiel dans sa portée. En DRT, nous disons que le même item construit une DRS et remplit son Univers. De plus, ce genre de confusion ou d'association des deux fonctions n'est pas un cas isolé en DRT, puisqu'il s'applique également à la représentation des groupes nominaux interprétés comme quantificateurs Universels: là aussi on suppose que le même item (tout) déclenche la construction d'une DRS et l'occupation de cette DRS par un Marqueur?.

Cette situation relativement simple est compliquée par deux questions que j'introduis ici sous une formulation simple, sans préjuger que cette formulation soit correcte, ni que les deux questions sont indépendantes :

1- L'usage des négatifs d'Univers comme items de polarité

2- L'interprétation des phrases contenant plus d'un négatif d'Univers.

\subsection{Négatifs d'Univers et items de polarité} de prototype. Le moindre ne peut pas se substituer à l'indéfini dans le contexte positif (28) :

(28) J'ai une chance

(29) * J'ai la moindre chance (36) :

(30) Je n'ai pas une chance

(31) Je n'ai pas la moindre chance

(32) Avez-vous une chance ?

(33) Avez-vous la moindre chance?

(34) Si vous avez une chance...

(35) Si vous avez la moindre chance...

(36) Tout homme qui a une chance doit essayer

(37) Tout homme qui a la moindre chance doit essayer

Ces contextes sont des contextes non-monotones; le remplacement de l'indéfini par le moindre ne produit pas de changement majeur de l'interprétation.

En laissant provisoirement de côté (30-31), nous pouvons observer que ce qui est vrai pour le moindre est également vrai pour les négatifs d'Univers dans un certain nombre de contextes :

(38) Avez-vous déjà vu quelque chose d'aussi beau? 
(39) Avez-vous jamais vu rien d'aussi beau ?

(40) Avez-vous déjà vu quelqu'un pleurer?

(41) Avez-vous jamais vu personne pleurer?

(42) Je me demande si quelqu'un a déjà dit une chose pareille

(43) Je me demande si personne a jamais rien dit de pareil (38-43) pour lesquelles aucune négation n'est à introduire dans la représentation. S'il est vrai, comme il est suggéré note 8 que ces configurations n'existent que dans les usages du français où ne est obligatoire avec un marqueur négatif, le problème est beaucoup moins difficile à résoudre qu'il ne pourrait sembler à première vue. Dans ce cas en effet, ou bien on se trouve dans un un usage à ne négatif obligatoire, et le négatif d'Univers est négatif si ne est présent, et item de polarité si ne est absent; ou bien on se trouve dans un usage 
sans ne négatif obligatoire, et tout usage de ces termes est négatif. Le point dépasse largement le cadre de ce travail et ne sera pas discuté plus avant.

47 La seconde conséquence, que nous aurons à discuter plus tard, est que cette ambiguïté pourrait être invoquée comme solution aux problèmes de compositionnalité, c'est-à-dire aux cas dans lesquels nous semblons avoir plus de négatifs dans la phrase que de négations dans la représentation.

\section{Phrases comportant plus d'un négatif d'Univers}

Une fois admis qu'un négatif pouvait être encodé comme une catégorie lexicale apparaissant plusieurs fois dans le domaine d'un même verbe, la possibilité est théoriquement ouverte d'avoir de nombreux négatifs dans une phrase simple.

Considérons quelques données du français9.

n'y a pas de contrainte grammaticale en français sur le nombre des négatifs d'Univers dans le domaine d'un verbe :

(44) Personne ne fait jamais rien nulle part

51 (44) est parfaitement grammatical, et contient quatre négatifs d'Univers pour un seul verbe.

\subsection{Phrases simples à sujet et objet négatifs}

(45) Personne n'aime personne

52 L'observation selon laquelle les phrases comportant deux négatifs d'Univers sont ambiguës a été faite de nombreuses fois dans la littérature (Cf. Muller 1991), le plus souvent à propos de phrases comportant un négatif d'Univers en sujet et en objet comme (45).

53 En DRT, les deux interprétations de la phrase sont correctement représentées par les DRS (46) et (47) : 


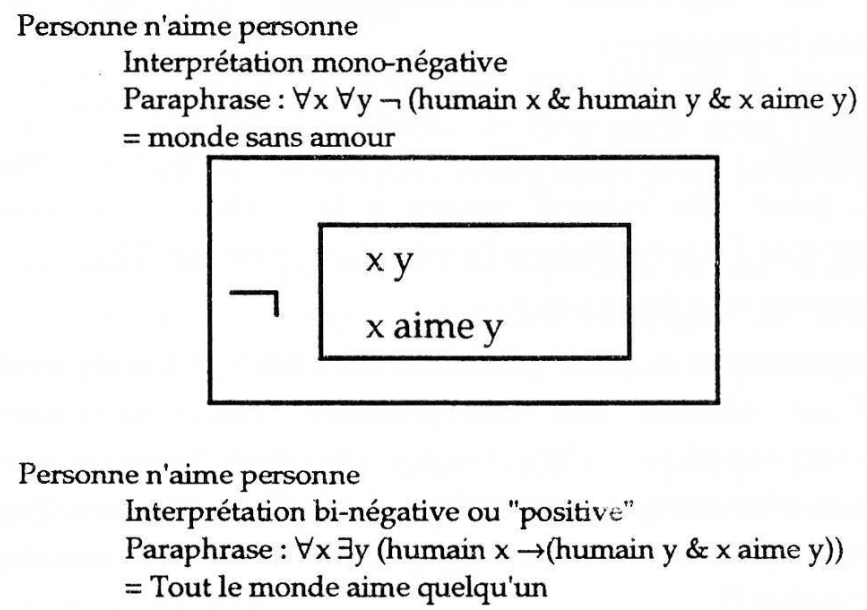

(47)

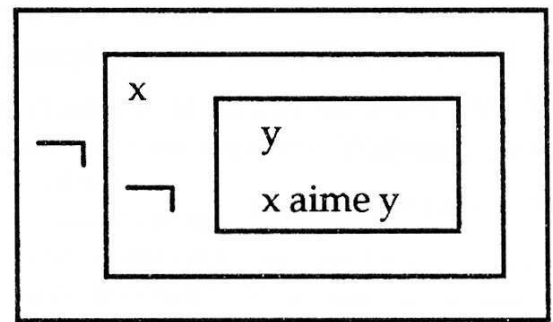

L'application de l'algorithme de Kamp et Reyle (Cf. 5 supra) à ces DRS fait des prédictions correctes sur les conditions de vérité correspondant à chacune des représentations.

On notera que ces interprétations sont logiquement contraires (elles ne peuvent être vraies toutes deux, mais il se peut qu'aucune des deux ne soit vraie). La même suite de mots peut par conséquent être en théorie utilisée pour faire une affirmation et pour contredire cette même affirmation. Il est possible d'imaginer des situations dans lesquelles cela pourrait effectivement se produire. Supposons par exemple que votre fils vous dise qu'il ne veut pas aller à telle soirée, et justifie sa position en disant (48) :

(48) Pourquoi y aller puisque personne n'aime personne, dans ce groupe ?

Il se peut que vous entrepreniez de le convaincre du contraire en utilisant la même phrase, comme en (49) :

(49) Mais c'est faux ! Personne n'aime personne : Alain adore Pierre, tu aimes Sarah et Jeanne...

57 Les locuteurs français disent le plus souvent que l'interprétation mono-négative est non marquée, tandis que l'interprétation bi-négative est plus difficile à obtenir, plus artificielle. En général, l'interprétation bi-négative semble être souvent utilisée pour contredire une autre phrase. Par exemple, si quelqu'un semble très déprimé, et vous dit (50) :

(50) Je suis triste, je n'aime rien Vous lui répondrez éventuellement (51) qui sélectionne l'interprétation bi-négative :

(51) Mais tu es fou. Personne n'aime rien; chacun aime quelque chose Cependant, il arrive que certaines configurations lexico-syntaxiques, pour des raisons tenant à la réalité visée, n'admettent naturellement que l'interprétation bi-négative, comme (52) :

(52) Personne n'est le fils de personne 
60 interprétation mono-négative, il faut imaginer qu'on applique (52) à une situation restreinte, soit par exemple : Dans cette entreprise, personne n'est le fils de personne. Dans ce cas seulement l'interprétation mono-négative «monde sans relation filiale » peut être vérifiable ${ }^{10}$.

61 Supposons que vous vouliez rendre un devoir à des étudiants, et que chacun d'eux ait fait au moins une faute ; étant donné que l'objectif d'un devoir est de ne pas faire de faute, la manière normale d'annoncer le résultat sera certainement (53) :

(53) Aucun étudiant n'a fait aucune faute

62 (53) a ici l'interprétation bi-négative, et il semble en général difficile d'obtenir l'interprétation mono-négative avec deux occurrences de aucun.

63 L'étude détaillée des conditions dans lesquelles une des interprétations sera préférée ou exclue n'est pas ici le point. La seule chose importante ici était de montrer que deux interprétations contraires existent pour la même suite de mots. Une théorie correcte devrait en rendre compte.

64 S'il semble bien s'agir de la même suite de mots, on pourrait douter qu'il s'agisse de la même structure syntaxique : deux structures différentes pourraient être impliquées, ou bien quelqu'espèce de topicalisation pourrait jouer un rôle dans l'existence de deux interprétations. En fait, il est vrai que l'on peut mécaniquement exclure l'interprétation mono-négative en accentuant fortement le négatif d'Univers en position d'objet direct, comme en (54):

(54) Personne n'aime // personne

La seule interprétation possible pour (54) est la bi-négative (47). Dans la mesure où il semble difficile de tirer parti pour l'instant de cette observation, nous ne pouvons que l'enregistrer pour la reconsidérer plus tard.

Supposer que chacune des interprétations est associée à une structure syntaxique propre semble une solution ad hoc; il parait préférable de ne retenir cette solution que si nous ne pouvons en trouver de meilleure.

Si nous excluons pour l'instant que deux structures syntaxiques distinctes sont en cause, la seule explication plausible réside dans l'usage de deux traitements différents pour la construction des interprétations à partir des items lexicaux.

Une manière simple de simuler la construction des représentations correctes (46) et (47) est proposée en (55) :

(55) Stratégie de traitement des négatifs d'Univers

Traiter la phrase de gauche à droite :

Si un négatif d'Univers est rencontré :

appliquer la stratégie générale pour les négatifs d'Univers (18) : créer une DRS négative, et traiter le constituant comme un indéfini inscrivant son Marqueur dans l'Univers de cette DRS.

Poursuivre le traitement de la Condition enchâssée :

Si un autre négatif d'Univers est rencontré, choisir l'une des deux solutions suivantes :

(a) Répéter la stratégie générale (18) :

sera alors produite une représentation bi-négative (récursivité)

(b) Traiter le constituant comme un indéfini inscrivant son Marqueur dans

l'Univers de la DRS négative précédemment créée : 
sera alors produite une représentation mono-négative avec deux Marqueurs dans son Univers (parasitisme).

La figure (1) est une repésentation schématique de cet algorithme :

Figure 1 : Traitement de deux négatifs d'Univers en position sujet et objet

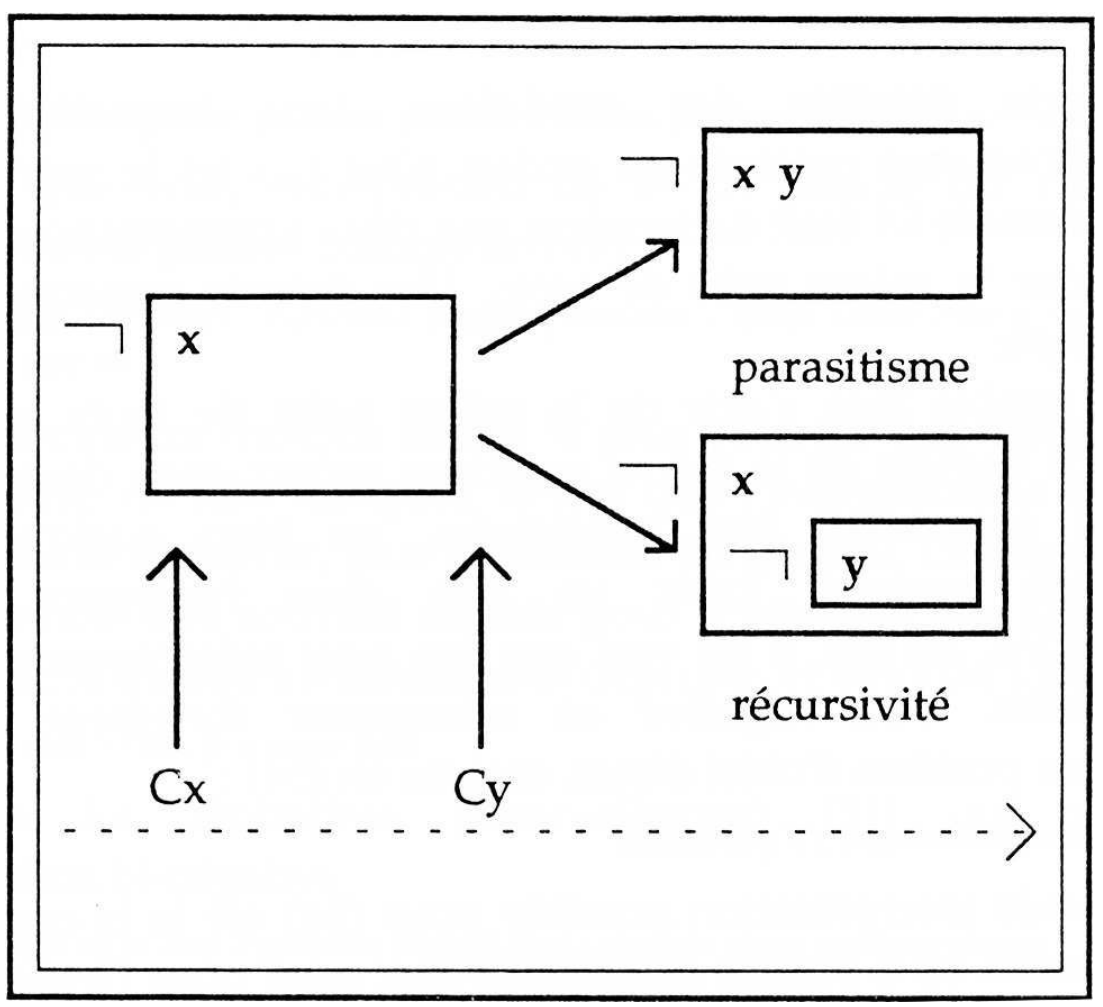

$70 C x$ et $C y$ représentent les deux négatifs d'Univers dans leur ordre d'occurrence ; $C x$ déclenche la construction d'une DRS négative dont l'Univers contient un Marqueur correspondant au constituant $x$; lors du traitement du second négatif d'Univers $c y$, ou bien l'univers de la DRS négative précédemment créée est utilisé comme site d'accueil pour le Marqueur correspondant au second constituant $y$ (parasitisme), ou bien une nouvelle DRS négative enchâssée est créée, dont l'Univers accueille le Marqueur y. (55) n'est qu'un schéma très général, tous les détails nécessaires à une implémentation effective ayant été laissés de côté.

Néanmoins, ce schéma général soulève plusieurs points intéressants :

Tout d'abord, l'ordre de traitement gauche-droite prédit la seule hiérarchie attestée pour les deux opérateurs négatifs de la version récursive: si $C x$ précède $C y$ dans la représentation, $x$ sera plus élevé que $y$ dans la hiérarchie de portée des négations ${ }^{11}$.

Ensuite, le schéma fait apparaître clairement le problème posé par l'interprétation du second terme dans le contexte créé par le premier ; il semble en effet que l'interprétation de ce terme puisse être sensible à ce contexte. Il apparaît dans ce schéma, grossièrement, que le second terme peut ne pas introduire de négation propre, pourvu qu'une négation d'Univers soit en cours de traitement.

Une première hypothèse qui vient à l'esprit pour traiter cette ambiguïté serait de la mettre en relation avec l'ambiguïté des négatifs d'Univers (négatif/item de polarité) signalée plus haut ${ }^{12}$. 


\subsection{Le second terme est-il un item de polarité ?}

Supposons que les négatifs d'Univers puissent s'interpréter comme négatifs, et comme items de polarité. Il y a au moins certains usages du français moderne dans lesquels l'ambiguïté est attestée en dehors de ces contextes.

Dans les phrases étudiées plus haut, la première occurrence de personne serait négative, et en tant que telle, créerait un contexte légitimant les items de polarité. Ce contexte légitimerait l'usage du second personne comme item de polarité, d'où l'interprétation mono-négative. Quant à l'interprétation bi-négative, elle reste naturellement autorisée en tout contexte, d'où l'interprétation bi-négative. L'ambiguïté se trouverait ainsi très simplement justifiée.

Je pense que l'idée est néanmoins peu plausible, et aurait en outre très peu de contenu explicatif.

On notera d'abord que la première occurrence de personne est sans aucun doute une occurrence qui légitime pour la position de la seconde les items de polarité. Le moindre est légitimé en position d'objet :

(56) Personne n'a la moindre idée

79 A cet égard, le négatif d'Univers initial personne a exactement le même effet que le négatif pas: dans les deux cas, le moindre est autorisé.

(57) Je n'ai pas la moindre idée

80 Mais le problème est justement que pas ne légitime, lui, aucune occurrence de personne en position d'objet qui pourrait être analysée comme un item de polarité. Considérons (58) :

(58) Je n'aime pas personne

81 La voie d'explication en cours d'examen prédit que dans (58) l'interprétation de personne comme item de polarité est légitime, et la phrase devrait signifier, au moins dans l'une de ses interprétations, la même chose que (59):

(59) Je n'aime pas quelqu'un

Or (58) ne peut pas avoir cette interprétation: (58) n'a qu'une interprétation, qui correspond à la version positive de (59), soit (60) :

(60) J'aime quelqu'un

La meilleur manière de prédire cela est d'assumer que (58) doit être représentée comme une phrase bi-négative, chacun des négatifs (pas et personne) étant responsable de l'introduction d'une négation dans la représentation.

84 Si donc nous voulions formuler effectivement l'analyse du second terme comme item de polarité, il nous faudrait soutenir que :

1- personne et pas créent des contextes qui légitiment l'usage des items de polarité.

2- l'interprétation item de polarité des négatifs d'Univers (personne, rien,...) est légitimée par un négatif d'Univers antérieur (personne, rien,...), mais non par un négatif antérieur.

Il me semble que si 2 doit être soutenu, aucun argument substantiel ne subsiste pour défendre l'analyse de la seconde occurrence comme item de polarité : si cela était vrai en (45), pourquoi ne le serait-ce pas en (58)?

Beaucoup d'autres arguments militent contre cette hypothèse. En français, il a été signalé précédemment ( $\$ 1.3$ ) que l'interprétation item de polarité n'est en général pas ouverte si ne est présent. Dans le cas de (45) ne est parfaitement légitime devant le verbe. Il nous 
faudrait donc abandonner une généralisation concernant le rapport forme-interprétation en français pour intégrer cette hypothèse. En outre, comme Muller $(1986,1991)$ l'a bien montré, beaucoup de langues n'ont pas d'usage indépendant des négatifs d'Univers comme items de polarité, alors qu'elles ont des interprétations mono-négatives correspondant à des structures à plusieurs négatifs d'Univers. Cela semble être la situation en anglais: selon May (1989), Nobody loves nobody a une interprétation mononégative, tout comme personne n'aime personne, alors qu'en anglais, il n'y a pas, semble-t-il, d'usage indépendant de nobody comme item de polarité.

Pour résumer les arguments, il semble que l'analyse comme item de polarité de la seconde occurrence, conduit à perdre plusieurs généralisations importantes, et reste une analyse ad hoc.

Avant d'introduire une autre théorie, il est utile de considérer ce qui se produit pour un nombre arbitraire de négations d'Univers dans le domaine d'un même verbe.

\subsection{Phrases avec plus de deux négatifs d'Univers} aux DRS (62) et (63) :

(62) Personne ne dit rien à personne Interprétation mono-négative

= mutisme absolu

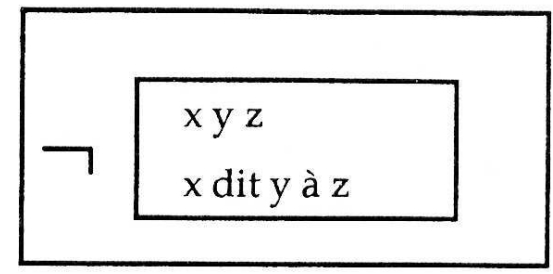

(63) Personne ne dit rien à personne Interprétation bi-négative

= Tout le monde dit quelque chose à quelqu'un

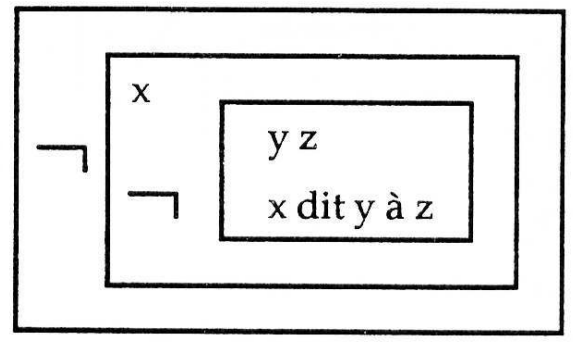

91 L'interprétation mono-négative (62) est celle qui se présente le plus facilement à l'esprit. Cependant, comme on a déjà eu l'occasion de le faire remarquer (Cf. 54), cette interprétation est exclue si l'un des négatifs d'Univers est fortement accentué.

(64) Personne // ne dit rien à personne

= interprétation bi-négative 
intéressant de comparer ces deux interprétations effectivement attestées, avec ce qui est possible en théorie, étant donné les deux opérations de base que nous avons dû postuler dans le cas plus simple, c'est-à-dire le parasitisme et la récursivité.

La figure 2 est une représentation schématique de toutes les combinaisons produites par un programme appliquant librement ces deux opérations en traitant les items de gauche à droite.

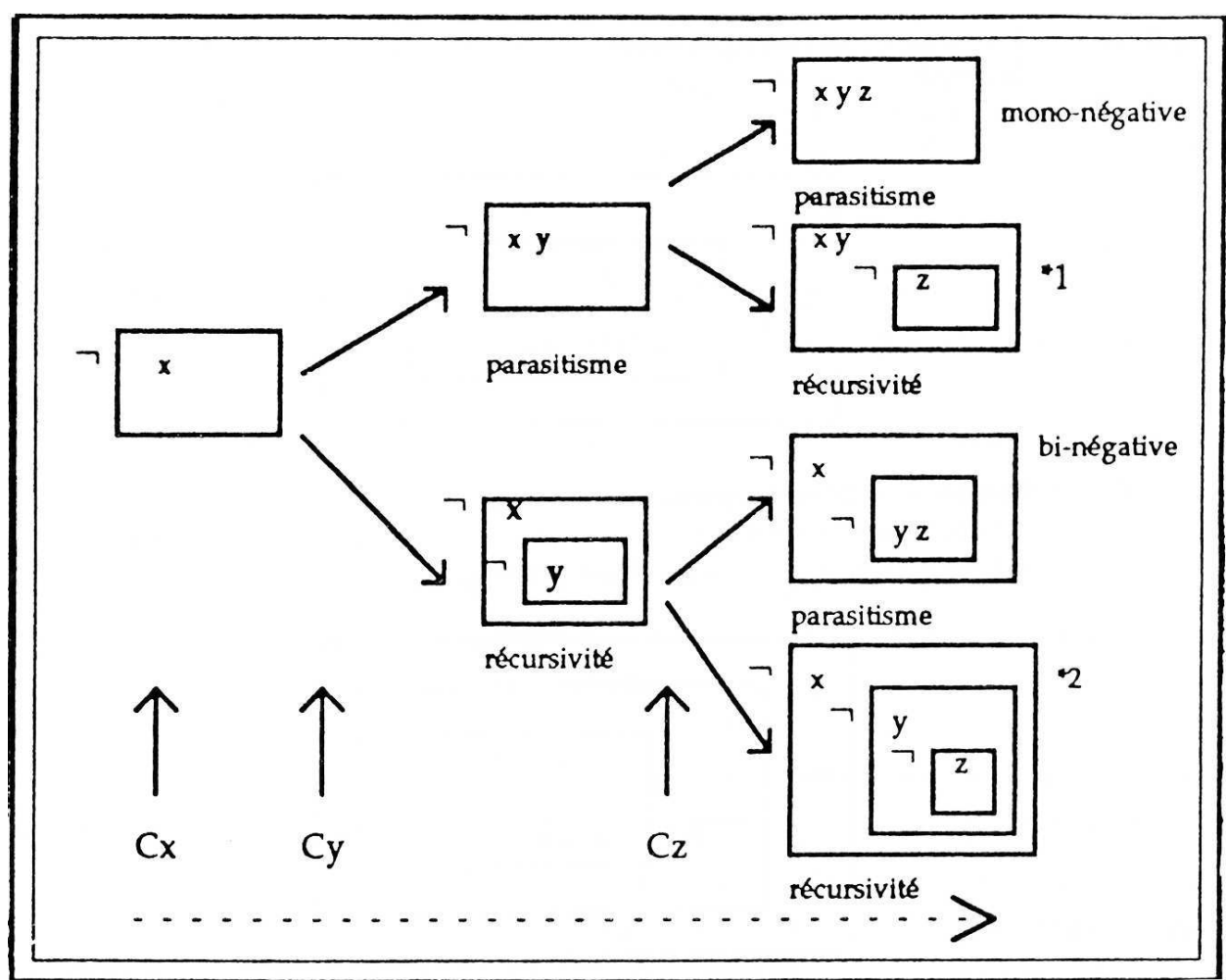

Seules deux des interprétations théoriques possibles correspondent effectivement à des interprétations que les locuteurs français acceptent d'associer à la suite (61). On doit noter qu'un programme n'aurait aucune espèce de difficulté à interpréter les représentations munies d'un astérisque. L'application de l'algorithme de vérification (5) qui fonctionne correctement pour toutes les interprétations attestées produit effectivement des interprétations pour $*_{1}$ et ${ }^{*} 2$ :

(65) Personne ne dit rien à personne interprétation bi-négative *1:

$=$ Pour tout $\mathrm{x}$ et tout $\mathrm{y}, \mathrm{il} \mathrm{y}$ a au moins un $\mathrm{z}$ tel que $\mathrm{x}$ dit $\mathrm{y}$ à $\mathrm{z}$.

En gros : pour tout humain, il existe au moins un humain à qui il dit tout

Exemple : Un homme n'a pas de secret pour sa femme. ${ }^{13}$

(66) Personne ne dit rien à personne

Interprétation tri-négative *2

= Pour tout $\mathrm{x}$, il $\mathrm{y}$ a au moins un $\mathrm{y}$ tel que pour tout $\mathrm{z}, \mathrm{x}$ ne dit pas $\mathrm{y}$ à $\mathrm{z}$

En gros : Tout le monde a au moins une chose qu'il ne dit à personne.

N'importe quel locuteur peut comprendre les paraphrases des représentations * 1 et *2, et peut imaginer des situations dans lesquelles elles sont vraies. Ce qu'un locuteur ne peut pas faire, c'est associer ces représentations à la suite linguistique considérée. En outre, cette impossibilité est forte: elle n'est pas similaire aux situations bien connues dans lesquelles un auto-enchâssement complexe de structures est à interpréter, comme les enchâssements relatifs ou les auto-enchâssements de négations classiques : 
(67) J'ai donné à la fille que le garçon aime un livre

(68) J'ai donné (à la fille (que le garçon (que le chien a mordu) admire)) un livre ${ }^{14}$

Dans ces cas dits de "limitation de performance ", le traitement des enchâssements complexes peut se révéler impossible dans un temps limité ; la structure alors ne reçoit pas d'interprétation. Si le locuteur se donne plus de temps, il réussit à obtenir l'interprétation correcte.

Dans le cas représenté par (65), la phrase est facile à interpréter, mais certaines des représentations théoriques sont strictement inaccessibles. Il s'agit donc d'une limite de compétence.

Si nous voulons obtenir toutes les représentations correctes au moyen d'un programme, deux opérations de base sont nécessaires, appelées ici parasitisme et récursivité. En outre, une limitation stricte doit être imposée au programme. Cette limitation pourrait être formulée comme en (69) :

(69) Limitation sur l'algorithme de représentation des négatifs d'Univers.

La profondeur maximale d'auto-enchâssement est 1 (voir *2).

Pas plus d'un Marqueur dans l'Univers le plus élevé d'un auto-enchâssement (voir *1).

La contrainte qui exclut ${ }^{*} 2$ est évidemment assimilable à une contrainte sur la complexité, puisqu'elle limite le degré d'auto-enchâssement possible ; celle qui exclut *1 peut aussi, quoique de manière moins évidente s'apparenter à une limite de complexité : l'idée serait que si deux Marqueurs sont dans l'Univers de la négation hiérarchiquement supérieure, la représentation est plus complexe que s'il y en a un seul.

Ce qu'on a appelé parasitisme, apparaît alors comme un moyen de limiter l'accroissement de complexité des représentations. Supposons que l'application de la récursivité conduise à dépasser le seuil de complexité, alors procéder par parasitisme a exactement pour effet de maintenir la structure dans les limites imposées.

Il ne s'agit naturellement que d'une des manières possible de « raconter l'histoire » de la figure (2). Cependant, il vaut la peine de noter qu'un système conçu sur ces bases semble produire les résultats corrects pour un nombre arbitraire de négatifs d'Univers. Considérons par exemple (70), qui contient quatre négatifs d'Univers :

(70) Personne ne dit jamais rien à personne

101 (70) n'a que deux représentations : ou bien le parasitisme s'applique constamment, auquel cas nous obtenons une représentation mono-négative, ou la récursivité s'applique pour la seconde occurrence, et ensuite, seul le parasitisme est possible.

Une autre observation intéressante est que, si nous suivons cette ligne d'explication, le premier écart lui-même, par rapport à la compositionnalité, peut paraître moins étrange. Nous avons effectivement des auto-enchâssements négatifs de ce type, mais aucun dont la profondeur soit supérieure à 1 , aucun dont l'Univers initial contienne plus d'un Marqueur. Nous observons donc une limite sur la complexité. Il est par conséquent plausible de considérer cet auto-enchâssement minimal lui-même comme une structure complexe, un seuil. Alors, l'application du parasitisme dès la seconde occurrence d'un négatif d'Univers peut apparaître comme déterminée par l'exigence de maintenir un bas degré de complexité pour ce type de représentation.

L'hypothèse qui vient à l'esprit est donc que le traitement des négatifs d'Univers est régulé par une limitation dynamique sur la complexité des représentations interprétables: un négatif d'Univers déclenche la construction d'une négation en 
inscrivant un Marqueur dans l'Univers de cette négation, à moins que cette action n'atteigne ou ne dépasse le seuil de complexité admissible; dans ce dernier cas, seule la dernière action est accomplie, et le négatif d'Univers "parasite» la négation précédemment introduite en y inscrivant son Marqueur ${ }^{15}$.

\section{BIBLIOGRAPHIE}

Barker, C., Dowty, D., (1992) Proceedings of Second Conference on Semantics and Linguistic Theory, Ohio State Working Papers in Linguistics.

Corblin, F., (1992) « Le traitement des complexes négatifs en DRT », in Actes du colloque ECCOS'92., Orsay, France, pp. 157-175.

Fauconnier, G., (1975) « Pragmatic Scales and Logical Structures », in Linguistic Inquiry VI-3, pp. 353-375.

—, (1977) « Polarité syntaxique et sémantique », in Linguisticae Investigationes I-1, pp. 1-38.

Gablay, D., Kempson, R. , (1992) « Natural-Language Content and Information Flow », ms.

Groenendijk, J., Jansen, T., Stockhof, M., (1984) Truth interpretation and information, Dordrecht, Foris.

Kamp, H., (1981) « A theory of truth and semantic representation », in GROENENDIJK 1984, pp. 1-41.

Kamp, H, Reyle, U., (1990) From Discourse to Logic, 2nd European Summer School, Leuwen.

Ladusaw, W.A. , (1992) « Expressing Negation », in BARKER 1992.

Laka, I., (1990) Negation in syntax: On the nature of functionnal categories and projection, Ph D. dissertation M.I.T.

Linebarger, M. , (1981) The grammar of Negative Polarity, Ph. D. Thessis, M.I.T.

May, R. , (1985) Logical form. Its structure and derivation, MIT press.

_, (1989) «Interpreting Logical Form », in Linguistics and Philosophy, 12, 4, pp. 386-437.

Muller, C. , (1986) La négation en français : syntaxe, sémantique et éléments de comparaison avec les autres langues romanes, Thèse d'Etat, Paris 7.

-, (1991) La négation en français, Droz, Genève.

Szabolcsi, A., Zwarts, F.

(1991) Unbounded Dependencies and Algebraic Semantics, Lectures Notes, Saarbrücken.

Van Benthem, J., (1989) « Polyadic Quantifier », in Linguistics and Philosophy, 13, 4, pp. 437-465.

Zwarts, F., (1992) The syntax and semantics of negative polarity, ms. Rijks Universiteit, Groningen. 


\section{NOTES}

1. D'autres traitements du problème existent auxquels nous ne pouvons ici rendre justice. Muller (1986) intègre les données dans une optique très différente quant à l'analyse des marqueurs négatifs du français. Ladusaw (1992) analyse le problème dans des termes voisins des nôtres et propose un traitement en termes de contraintes structurales sur l'expression de la négation. La position de Laka (1990) est discutée dans cet article de Ladusaw.

2. Voir Corblin (1992) pour une présentation plus détaillée. DRT abrège Théorie des Représentations du Discours; DRS abrège structure de représentation de représentation de discours.

3. Afin de ne pas introduire de confusion entre l'usage technique de marqueur en DRT, pour marqueur de référence, (angl. marker), et l'usage non technique de marqueur pour l'item lexical porteur d'une interprétation (angl. trigger), le terme technique sera toujours écrit avec une initiale majuscule.

4. Condition, avec initiale majuscule distinguera l'usage technique du terme en DRT.

5. Pour une hypothèse opposée, voir notamment la thèse de Muller (86) et Muller (91).

6. Il y a comme on le sait, une hypothèse beaucoup plus élaborée sur le statut de ne et pas en français, celle du discordantiel et du forclusif, due à Damourette et Pichon. Comme l'a rappelé M. Arrivé dans sa communication, la thèse des auteurs est en fait qu'il n'y a pas de négation en français, la négation étant remplacée par la combinaison du discordantiel et du forclusif.

7. Cf. Kamp (1981), Kamp et Reyle (1990).

8. Cela signifie que l'usage des négatifs d'Univers comme termes de polarité n'existe guère dans les usages du français où ne n'est pas utilisé dans les phrases négatives. On pourrait suspecter qu'il y a là une relation causale. En tous cas, il est tentant d'opposer deux situations idéales :1) ne est obligatoire avec l'usage d'un marqueur négatif ; on peut alors distinguer l'usage négatif (avec $n e$ ) et l'usage comme item de polarité (sans ne) d'un même item. 2) ne n'apparaît pas avec les marqueurs négatifs; on ne peut alors distinguer les deux usages.

9. Nous considérons essentiellement le français écrit correct. En conséquence, le clitique préverbal ne est toujours représenté dans les exemples. En l'absence de stipulation contraire, ce qui est dit en matière de grammaticalité et d'interprétation des exemples reste vrai pour les usages du français sans ne négatif.

10. Des exemples tels que: (a) Rien ne naît de rien; (b) Personne ne travaille pour rien m'ont été proposés respectivement par D. Godard (c. p.) et David Gaatone (discussion). On pourrait y ajouter $(c)$ : On n'a rien sans rien.

Ces exemples n'admettent que l'interprétation bi-négative: Tout naît de quelque chose; Tout le monde travaille pour quelque chose. Comme me l'a signalé un participant au colloque, ces exemples ont ceci de particulier que rien après certaines prépositions peut apparaître mais ne légitime pas à lui seul la présence de ne devant le verbe : $(d)$ *'L'homme ne naît de rien; (e) *Pierre ne travaille pour rien; $(f)$ *Pierre ne travaille sans rien. Rien ne peut pas par conséquent être analysé dans ces exemples comme un négatif d'univers dans le domaine syntaxique du verbe. Il n'en a ni les propriétés syntaxiques (occurrence facultative de ne préverbal), ni sémantiques (interprétation mono-négative en combinaison avec un négatif d'univers dans le domaine du verbe).

11. D'après May (1989), les phrases parallèles de l'anglais (Nobody loves nobody) peuvent être interprétées avec l'une ou l'autre des hiérarchies de portée. En français, l'interprétation avec le sujet dans la portée de l'objet (Tout le monde est aimé de quelqu'un) n'existe pas.

12. L'idée a souvent été mentionnée dans la littérature sur le français. Voir Muller $(1986,1991)$ pour une revue. 
13. Une autre interprétation bi-négative n'est pas totalement exclue $:$ si $\mathrm{x}$ dit $\mathrm{y}, \mathrm{x}$ dit $\mathrm{y}$ à un $\mathrm{z}$ au moins, c'est-à-dire en substance Personne ne parle tout seul. Ce type d'interprétation ne peut pas être discuté dans le cadre limité de cet article.

14. Je remercie Jonathan Ginzburg pour cet exemple.

15. Comme indiqué au début de l'article, nous devons nous limiter ici à une présentation générale. Une formulation plus détaillée, assortie d'arguments pour défendre cette approche est en cours de préparation (Multiple Negation Processing, ms.). Parmi les points empiriques importants qui n'ont pas pu être abordés ici : le statut du négatif pas par rapport aux négatifs d'Univers; l'étude des contextes à interprétation mono-négative exclue ; l'étude des contextes à interprétation bi-négative exclue ; l'étude des interprétations mentionnées dans la note 13.

\section{RÉSUMÉS}

Cet article examine l'expression de la négation dans les phrases françaises de type: Personne n'aime personne. En premier lieu, on introduit dans le cadre de la Théorie des Représentations du Discours (DRT), une distinction entre deux sortes d'expressions négatives: Négatifs (pas), et Négatifs d'Univers (rien, personne, aucun $N$, ...). Ensuite, on examine plus particulièrement l'ambiguïté bien connue des phrases à négatifs multiples telles que Personne n'aime personne, ou Personne ne dit rien à personne. Pour beaucoup d'auteurs, ces phrases semblent constituer une «abrogation de la compositionnalité » (May 1989), puisqu'il n'y a pas de correspondance biunivoque entre les négatifs dans la phrase et les négations dans la représentation sémantique. L'étude des phrases françaises comportant plus de deux négatifs d'Univers conduit à l'observation suivante : on obtient une représentation correcte en appliquant un algorithme de construction gauche-droite, lequel maintient la représentation sémantique en deçà d'un seuil de complexité. Ce résultat est obtenu par parasitisme: l'expression potentiellement négative est traitée comme un indéfini dans le domaine de la négation en cours.

This paper considers the relationship between negative triggers and negation in French sentences like Personne n'aime personne. Firstly a distinction between two kinds of negative triggers is proposed in the framework of Discourse Representation Theory: Negatives (pas), and Universe negatives (rien, personne, aucun $N, \ldots$ ). Then the well known ambiguity of multi-negative sentences like Personne n'aime personne or Personne ne dit rien à personne is revisited in some details. For many authors those sentence look like an, «abrogation of compositionality » (May, 1989), because in some readings we do not get a one to one correspondence between the negative triggers in the sentence and the negations in the semantic representation. The study of French sentences whith more than two Universe negatives leads to the following observation: we get a correct representation assuming a left-right construction algorithm, which keeps the resulting semantic representation below a very low degree of complexity; this result is achieved by parasiting a negation in process, instead of introducing an auto-embedded negation.

\section{AUTEUR}

\section{FRANCIS CORBLIN}

UHB Rennes 2, CNRS URA 1028 